\title{
Investigating the role of plasma topography on chromospheric oscillations observed by TRACE
}

\author{
S. W. McIntosh ${ }^{1}$, B. Fleck ${ }^{1}$, and P. G. Judge ${ }^{2}$ \\ ${ }^{1}$ European Space Agency, Research and Scientific Support Department, NASA Goddard Space Flight Center, \\ Greenbelt, MD 20772, USA \\ ${ }^{2}$ High Altitude Observatory, National Center for Atmospheric Research ${ }^{\star}$, PO Box 3000, Boulder, CO 80307, USA
}

Received 10 February 2003 / Accepted 15 April 2003

\begin{abstract}
We present the results of an investigation into the interaction of the topographic structure of the solar chromospheric plasma with the wave modes manifesting themselves in the UV continua formed there. We show that there is a distinct correlation between the inferred plasma topography, the phase-differences between and suppression of oscillations in different levels of the solar atmosphere. We interpret these factors as evidence of interaction between the oscillations and the extended magnetic "canopy". This work is based on the analysis of joint observations made by the Solar and Heliospheric Observatory (SOHO) spacecraft and the Transition Region and Coronal Explorer (TRACE).
\end{abstract}

Key words. Sun: chromosphere - Sun: oscillations - Sun: UV radiation

\section{Introduction}

The question still exists as to the exact evolutionary path, from generation to extinction, of the ubiquitous oscillations observed in the quiet solar photosphere and chromosphere. The underlying goal of this quest is to assess whether or not there is a sufficient wave flux to supply energy, through some as yet unidentified mechanism, to the ambient chromospheric and coronal plasmas. Recent publications, in a concerted bid to answer this question, have resulted in the focus being placed on the understanding and analysis of multi-spacecraft, multi-wavelength, observations of chromospheric oscillations (see, e.g., Judge et al. 2001, hereafter JTW).

In this Paper we will take a closer look at one subset of those data, described in JTW, from the Solar and Heliospheric Observatory (Fleck et al. 1995, SOHO) Joint Observing Plan (JOP72). Specifically, we will study the time-series of the Ultraviolet (UV) continua (at 1550, 1600 and $1700 \AA$ ) acquired by the Transition Region and Coronal Explorer (Handy et al. 1999, TRACE) and the longitudinal magnetic field measured by the Michelson Doppler Imager (Scherrer et al. 1995, MDI) of SOHO on February 26, 1999 between 23:00 and 00:00 UT on February 27, 1999.

The work presented in McIntosh et al. (2001) and McIntosh \& Judge (2001) utilised SOHO/SUMER (Wilhelm et al. 1995)

Send offprint requests to: $\mathrm{S}$. W. McIntosh, e-mail: scott@esa.nascom.nasa.gov

* The National Center for Atmospheric Research is sponsored by the National Science Foundation. time-series data to investigate the relationship between the extrapolated plasma topographies and the effect on the observed spectroscopic signatures. This work was limited to one spatial dimension, that of the SUMER slit. We demonstrate that there is a clear extension of this previous work to more than the one spatial dimension presented by the SUMER spectrometer. That is, we show that there is a direct correlation between reduced oscillatory power in the TRACE UV continua and the transition to a low plasma- $\beta$ (ratio of gas pressure to magnetic pressure) regime in the formation heights of the TRACE continua. Further, we suggest that the TRACE time-series data can be used as a diagnostic of the plasma topography and conditions in the mid-chromosphere through the signatures of the wavemodes present; a form of "chromo-seismology", see Sect. 2.1.

\section{Analysis}

We analyse a $100^{\prime \prime} \times 200^{\prime \prime}$ sub-region of the JOP72 TRACE data of February 26, 1999 (see discussion in JTW). The observations were made of a near disc-center region of dimension $128^{\prime \prime} \times 320^{\prime \prime}$ with $15 \mathrm{~s}$ between frames of the same bandpass. We have employed the data reduction method outlined in Sect. 2 of Krijger et al. (2001) to investigate the time-series of the three UV TRACE bandpasses at $1550 \AA$, $1600 \AA$ and $1700 \AA$. This method allows us to form datacubes for the TRACE and MDI time-series, $D_{\lambda_{i}}(x, y, t)$, with each frame coaligned with the previous one, until all are coaligned to that at $t=0$ with sub-pixel accuracy (Tarbell 2002, Private Communication, employing the TRACE Interactive 


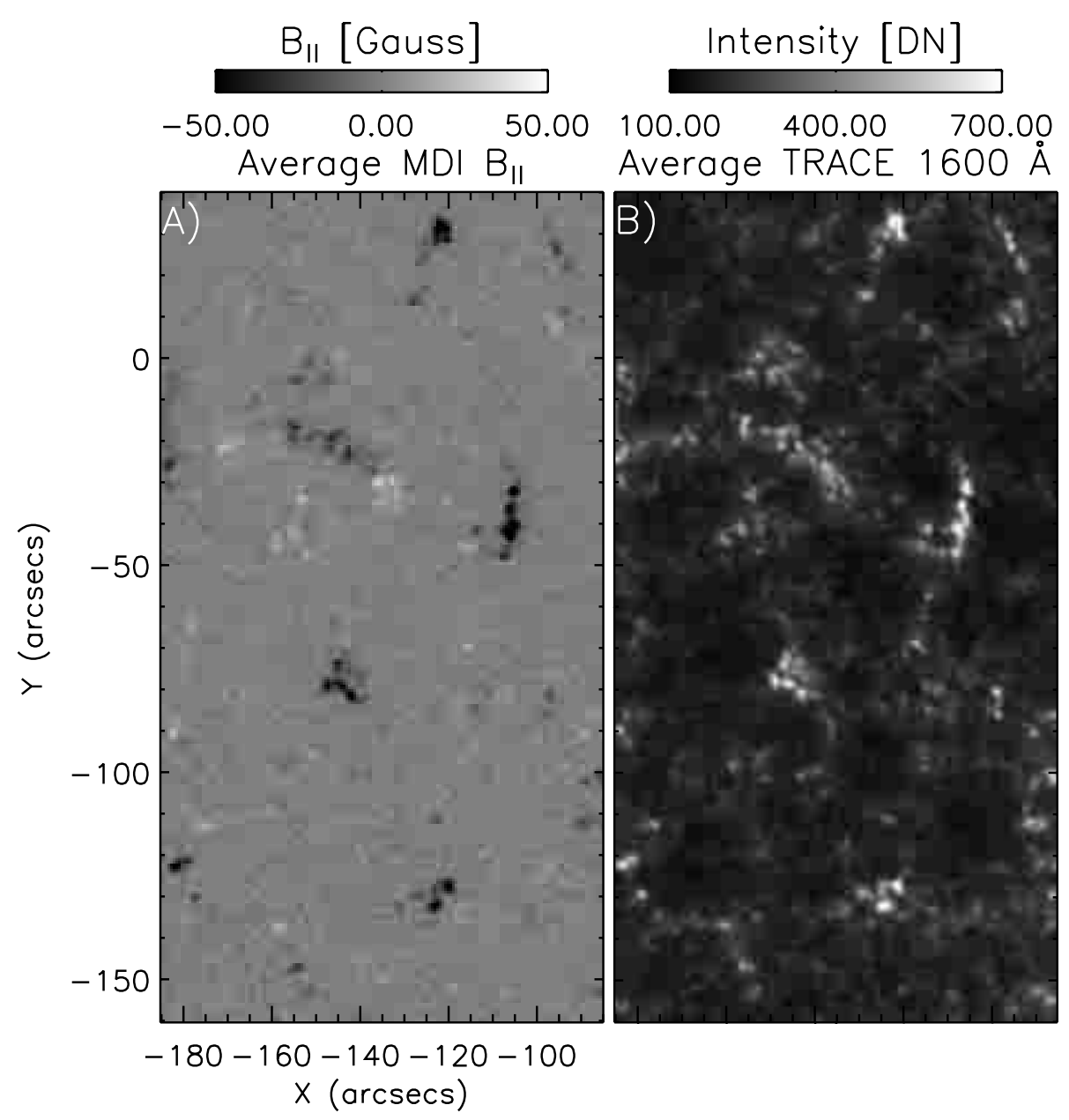

Fig. 1. Coaligned context SOHO/MDI (left panel; $B_{\|}$) and TRACE (right panel; $1600 \AA$ A) images from JOP72 data-cubes of February $26,1999$. These images represent of the averages of one hour of time-series, 60 and 240 frames respectively.

Data Language routine "tr_get_disp_2d.pro"). This image correlation process ensures that the data-cubes are as "stable" as possible to the effects of solar rotation. The need to have each of these data-cubes aligned with one another required that the initial TRACE and MDI frames must be carefully correlated to one another ${ }^{1}$. Figure 1 provides some context for the observations through the temporal averages of the MDI line-of-sight magnetic field $B_{\|}$and TRACE (the $1600 \AA$ bandpass) timeseries and demonstrates that they are indeed well coaligned.

The critical component of the analysis presented by McIntosh et al. (2001) was the correlation between continuum signal formation height and the region of the atmosphere where the plasma- $\beta$ is of order unity. This correlation was represented by a considerable drop in the integrated oscillatory power in the $3-8 \mathrm{mHz}$ frequency band when the SUMER signal was formed in the low $\beta$ regime. So, to draw comparison between the twodimensional TRACE data and the one-dimensional SUMER data we must form an extrapolation of the underlying magnetic field. Since the coaligned MDI magnetograms show very little signature of emerging flux over the hour of the time-series,

\footnotetext{
${ }^{1}$ This coalignment is slightly more involved than normal because the SOHO spacecraft was rolled by $119.256^{\circ}$ from the solar NorthSouth line.
}

we can assume that the region under observation was very static. Therefore, in an effort to build a picture of the chromospheric plasma topography we employ the simplest approach to extrapolate the magnetic field, a potential extrapolation ${ }^{2}$, of the time-averaged MDI $B_{\|}$. Once the three dimensional field $B(x, y, z)$ is extrapolated from $B_{\|}(x, y, 0)$ we compute the magnetic pressure $P_{\mathrm{B}}(x, y, z)=B_{\|}(x, y, z)^{2} / 8 \pi$ and the plasma- $\beta$ ( $\beta \equiv P_{\mathrm{g}} / P_{\mathrm{B}}$; where $P_{\mathrm{g}}$ is the model gas pressure). We make use of the Vernazza et al. (1981, VAL3C) model values of $P_{\mathrm{g}}$ interpolated onto the same vertical scale as that of the magnetic field extrapolation.

To search for locations where the oscillatory power drops in the TRACE bandpasses they are further decomposed, using a Fast Fourier Transform at each spatial pixel, so that we have

$\hat{f}_{\lambda_{i}}(x, y, v)=\frac{1}{\sqrt{2 \pi}} \int_{-\infty}^{\infty} D_{\lambda_{i}}(x, y, t) \mathrm{e}^{-i v t} \mathrm{~d} t$,

where $\hat{f}$ indicates that the new cube is a complex quantity. We have exchanged the time coordinate for a frequency

\footnotetext{
${ }^{2}$ While more sophisticated extrapolations, using magneto-static equilibrium calculations (as done by Metcalf et al. 1995), for example, are appealing, not enough is known concerning the gas thermodynamics to make them useful, in this context.
} 

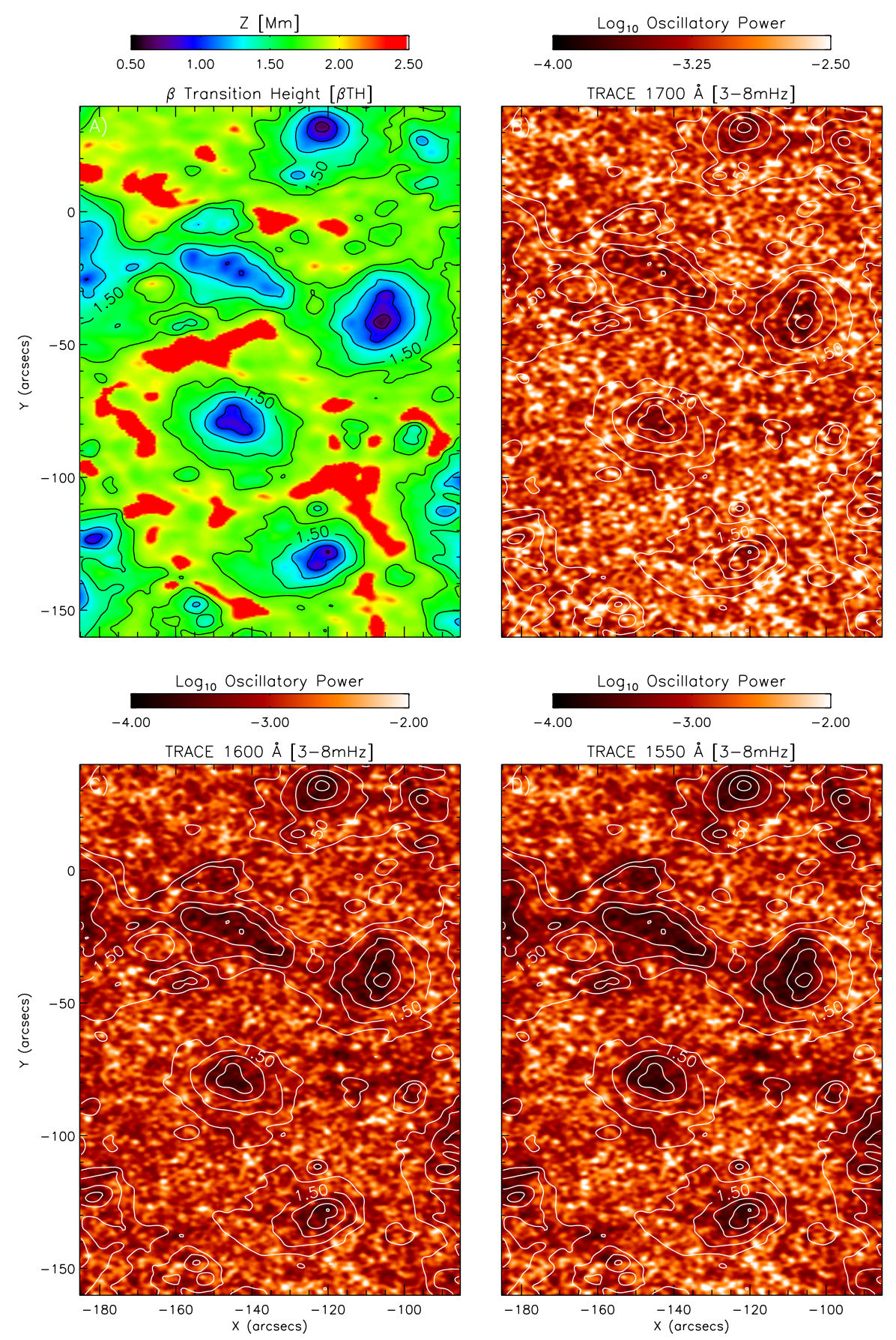

Fig. 2. In panel A we show the spatial variation in the altitudes at which the plasma- $\beta$ is of order unity in the TRACE field of view, the $\beta$ TH. We have included solid contours for reference and each is separated by $0.25 \mathrm{Mm}$. panels B through D show one example of the integrated oscillatory power in the 3-8 mHz band of the 1700, 1600 and $1550 \AA$ TRACE UV bandpasses respectively. On each panel we have overplotted the contours of panel A. Note the clear correlation between the reduced power and the contours.

coordinate and we have created equivalent cubes, $\hat{f}_{\lambda_{i}}(x, y, v)$, in frequency $v$ (units: $\mathrm{mHz}$ ). From these new cubes, and for comparison with the results of McIntosh et al. (2001), we can now construct maps of integrated oscillatory (Fourier) power in the $3-8 \mathrm{mHz}$ range.

Now, it is straightforward to estimate the height at which the plasma- $\beta$ is of order unity, five say, in the two-dimensional TRACE field-of-view (FOV). This height we call, for the sake of brevity, the $\beta$ transition height, or $\beta \mathrm{TH}$. In panel A of Fig. 2 we show the colour-coded spatial variation of the $\beta \mathrm{TH}$. As a visual aid we have added contours with a spacing of $0.25 \mathrm{Mm}$. The presence of the network elements, where $\beta \mathrm{TH}$ is blueviolet in colour, is perhaps the most striking feature. We can also get a feeling, albeit a very rough one given that this is from a potential field extrapolation, about the topographical form of the chromospheric "canopy" field and its connectivity. In the inter-network regions of panel $\mathrm{A}$ it is clear that the $\beta \mathrm{TH}$ is far higher than that in the network regions. In the other panels of 


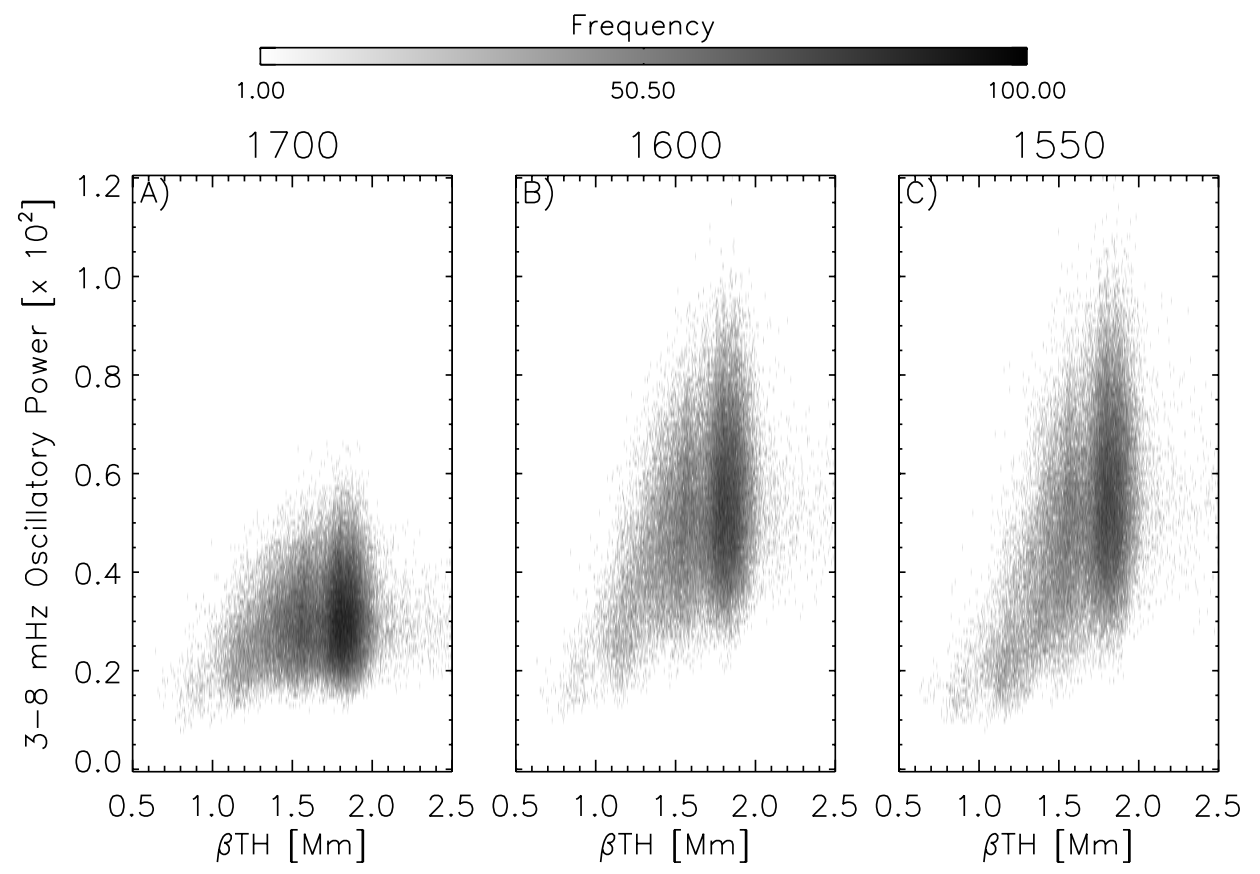

Fig. 3. The two-dimensional histograms of TRACE oscillatory power and $\beta$ TH derived from panels B through D of Fig. 1. The colour scale in each panel indicates the number of image pixels with that particular combination of oscillatory power and $\beta \mathrm{TH}$. We see that, in each panel, there are two distinct correlations present, the "tail" to the lower-left indicating the correlation of low oscillatory power and low $\beta \mathrm{TH}$ and the broad power correlation of the "canopy" at a $\beta \mathrm{TH}$ of around $1.75 \mathrm{Mm}$.

Fig. 2, B through D, we have included the maps of $3-8 \mathrm{mHz}$ power for the three TRACE UV bandpasses with the same $\beta \mathrm{TH}$ contours overplotted. Obviously, there is a striking correspondence between the $\beta \mathrm{TH}$ contours and the dearth of oscillatory power and that is far extended from the network elements, cf. panel B of Fig. 1 (Krijger et al. 2001 reported the drop of the 3-8 $\mathrm{mHz}$ power and called this a "network shadow"; quite different from the "shadow" defined by McIntosh \& Judge 2001). The clear extension of the drop beyond the the network element makes it all the more likely that the drop in signal is more than just an issue related to regions where there is a large intensity contrast in that particular continuum bandpass.

These TRACE continua are formed at heights of 400$700 \mathrm{~km}$ above $\tau_{500}=1$ (JTW), we see that the $\beta$ TH contours corresponding to significantly reduced oscillatory power in the TRACE continua are at $1.0 \mathrm{Mm}$ or less. In an effort to quantify this correspondence we show, in Fig. 3, the two-dimensional correlation histogram of oscillatory power and $\beta \mathrm{TH}$ for each of the three TRACE bandpasses. There are two correlations that are clearly present in each of the histograms:

- A vertical span at a range of oscillatory powers at a $\beta \mathrm{TH}$ of $1.75 \mathrm{Mm}$, corresponding to the "canopy" heights in panel A of Fig. 2.

- A "tail" increasing linearly from the lower-left of the histogram.

The latter indicates that the smallest oscillatory powers are indeed related to the regions where the $\beta \mathrm{TH}$ is lowest.

There is an extension of the TRACE continua analysis that can help us to differentiate between the correlation between "canopy" interactions with oscillations (i.e., $\beta \mathrm{TH}$ and oscillatory power suppression) and the suppression of oscillations in regions of magnetic plage (see, e.g., Title et al. 1992). In Fig. 4, we have duplicated the TRACE UV continuum analysis for the concurrent MDI filtergram time-series in $B_{\|}$, the line-of-sight Doppler velocity $V_{\|}$and continuum intensity $I$; all of which are formed significantly lower $(\sim 0.2 \mathrm{Mm})$ than the TRACE UV continua ${ }^{3}$. The rationale being that, at these lower formation heights, we should see very little, or no, oscillatory power suppression in $V_{\|}$and $I$ except in regions where the $\beta \mathrm{TH}$ gets low enough, i.e. in concentrated, strong, regions of network or plage magnetic fields, not the extended regions shown in Fig. 2. Panels B through D of Fig. 4 show, again, the integrated power in the $3-8 \mathrm{mHz}$ range for the three MDI filtergram time-series. Other than the dark regions of low power in panel B (such deficits of $B_{\|}$Fourier power have been discussed previously in the literature, see, e.g., Jain \& Haber 2002), a cursory look at panels $\mathrm{C}$ and $\mathrm{D}$ shows the reader little, or no, correlation to the overplotted $\beta \mathrm{TH}$ contours. However, careful examination of panel D shows that the lowest oscillatory powers are located in the very core of the network elements; the darkest regions in panel A. The localisation of these oscillatory power minima in the network element cores is consistent with the results found in the TRACE UV continua analysis. This adds further weight to the assertion that the observed oscillatory power suppression is related to the topographic structure of the plasma as interpreted through the $\beta \mathrm{TH}$.

Using the evidence presented in Figs. 2, 3 and 4 we deduce that the reduction in the oscillatory power of the TRACE continua, MDI $I$ continuum and their close correlation to the

\footnotetext{
3 These time-series, from the MDI high resolution field of view, have a cadence of one per minute.
} 

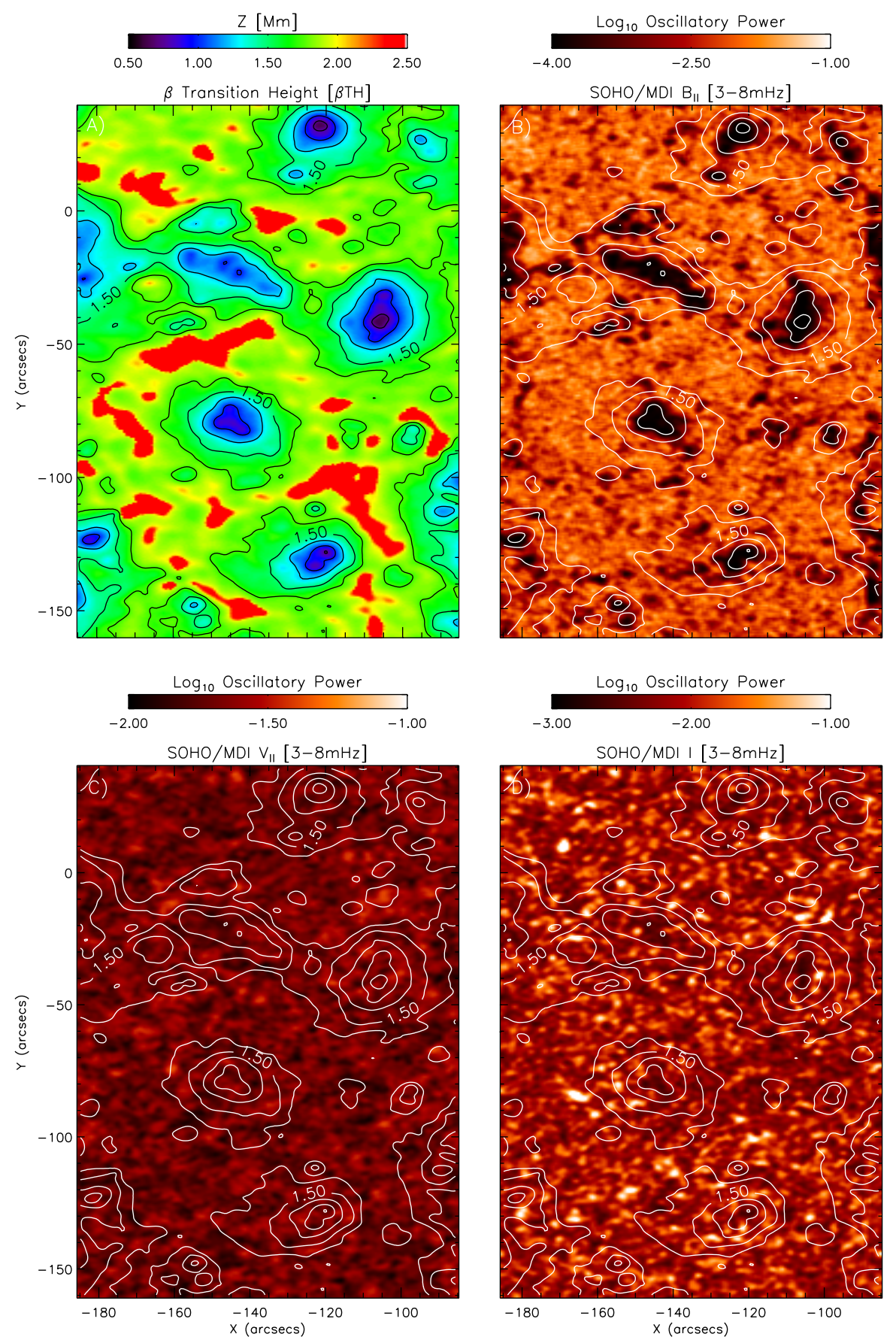

Fig. 4. In panel $\mathrm{A}$ we show the spatial variation in the altitudes at which the plasma- $\beta$ is of order unity in the SOHO/MDI field of view, the $\beta \mathrm{TH}$. We have included solid contours for reference and each is separated by $0.25 \mathrm{Mm}$. panels B through D show one example of the integrated oscillatory power in the $3-8 \mathrm{mHz}$ band of the $B_{\|}, V_{\|}$and continuum intensity $(I)$ MDI filtergrams respectively. These filtergrams are formed significantly lower in the atmosphere than those of Fig. $2(\sim 0.2 \mathrm{Mm})$. On each panel we have overplotted the contours of panel A. Careful inspection of panel D shows that the only significant drops in oscillatory power occur in the very darkest, deepest, regions of the $\beta \mathrm{TH}$.

structure of the $\beta \mathrm{TH}$ is the two-dimensional analogue of the effect reported by McIntosh et al. (2001).

\subsection{Bandpass phase-differences and plasma topography}

An obvious extension of the work discussed in this paper is to study the spatial variation of the phase-difference between the three TRACE bandpasses at as high a spatial resolution as possible. The investigation of phase-differences in TRACE, SUMER and ground based observations of chromospheric oscillations has been performed before (see, e.g., Lites et al. 1982; Deubner \& Fleck 1990; Krijger et al. 2001, JTW and many more references therein). These previous analyses spatially summed phase-spectra and sought only to differentiate between network and inter-network regions (ensuring a large 
signal-to-noise level), not to investigate the full range of spatial features. Here, we compute the TRACE bandpass phasedifferences (as outlined in Sect. 3 of Krijger et al. 2001 ${ }^{4}$ ) directly from the full spatial resolution complex frequency cube of Eq. (1). To compute the phase-difference spectrum we must first compute the cross-power spectrum $\hat{C}_{i, j}(x, y, v)$ for any two of the three bandpasses $i$ and $j$, say. This is simply defined by

$\hat{C}_{i j}=\hat{f}_{\lambda_{i}} * \hat{f}_{\lambda_{j}}^{\dagger}$

where $\hat{f}^{\dagger}$ is the complex conjugate of $\hat{f}$. The phase-difference spectrum $\Delta \phi_{i j}(x, y, v)$ is given by the argument $\left(\Delta \phi_{i j} \in[-\pi, \pi]\right)$ of $\hat{C}_{i j}$, i.e.,

$\Delta \phi_{i j}(x, y, v)=\tan ^{-1}\left(\frac{\mathfrak{J}\left\{\hat{C}_{i j}(x, y, v)\right\}}{\mathfrak{R}\left\{\hat{C}_{i j}(x, y, v)\right\}}\right)$.

We compute the phase-difference spectra for the three bandpass

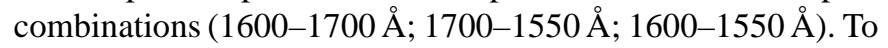
characterise the phase-difference behaviour spatially we have to measure the phase-difference change over a limited frequency range and one approach is to estimate/fit the gradient $\left(M_{\Delta \phi}=\mathrm{d}\left(\Delta \phi_{i j}\right) / \mathrm{d} v\right)$ of the phase-difference spectrum as a function of frequency. This gradient, $M_{\Delta \phi}$, is effectively proportional to the difference in bandpass formation heights $\left(\Delta z_{i j}\right)$ and the reciprocal of the phase-speed ( $\left.V_{\text {phase }}\right)$ of any perturbation present there, $M_{\Delta \phi} \approx \Delta z_{i j} / V_{\text {phase }}$. However, the task of fitting $M_{\Delta \phi}$ cannot be performed at the full TRACE spatial resolution $\left(0.5^{\prime \prime}\right)$ as the statistical variation (scatter due to noise) of phase-difference points at each spatial position does not allow for the accurate assessment of $M_{\Delta \phi}$ (see below and Fig. 5 for an example). So, we must reduce the spatial resolution by a reasonable amount and specify a new spatial grid size, say $5^{\prime \prime} \times 5^{\prime \prime}$ (or $10 \times 10$ TRACE pixels).

To investigate the spatial variation of $M_{\Delta \phi}$, we form a scatter plot of $\Delta \phi$ versus $v$ for each pixel in the $10 \times 10$ spatial pixel region. Additionally, each of these points has a weight (given by its corresponding cross-spectrum power values) and we use these weights to perform a linear least-squares fit to the scatter plot between 3 and $8 \mathrm{mHz}$. A sample fit of $M_{\Delta \phi}$ from the scatter plot is illustrated in Fig. 5 for a typical internetwork aggregate pixel. The dots indicate the scatter in the $\Delta \phi_{i j}, v$ space (notice the tight behaviour and growth from about $3-10 \mathrm{mHz}$ and the break to almost random phase-differences beyond $15 \mathrm{mHz}$ ) and the size indicates the magnitude of the cross-power (largest have the highest cross-powers), or the weights of the fit. This example fit has a $M_{\Delta \phi}$ of $7.4 \pm 0.8^{\circ} \mathrm{mHz}^{-1}$. A more in-depth discussion of this procedure, and the associated fitting error, can be found in McIntosh et al. (2003).

Performing this analysis for each aggregate pixel of the three bandpass combinations we can construct the panels of Fig. 6. Again, for reference, in panel A, we show the $\beta \mathrm{TH}$ map and its contours. In panels B through D we show the spatial dependence of $M_{\Delta \phi}$ for each of the bandpass pairs; $1700-1600 \AA$,

\footnotetext{
${ }^{4}$ This analysis includes the incorporation of the fact that the three bandpass images are not taken simultaneously, thus, the phasedifference spectra require a frequency-dependent shift to be calculated and applied.
}

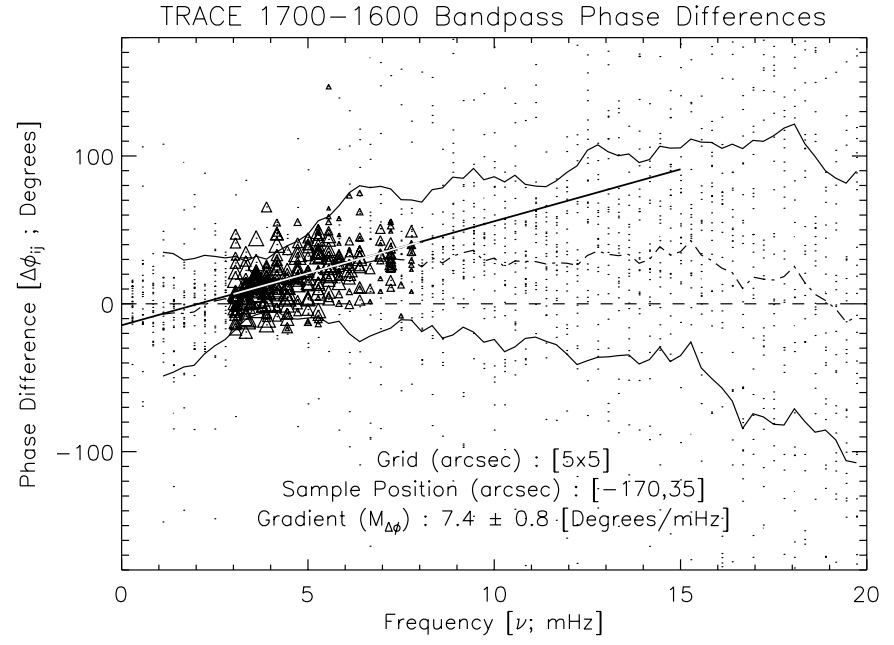

Fig. 5. Sample linear fit of the phase-difference gradient, $M_{\Delta \phi}$, between 3 and $8 \mathrm{mHz}$ at position coordinates $[-170,35]$ and a $5^{\prime \prime} \times 5^{\prime \prime}$ region around that point for the $1700-1600 \AA$ bandpass pair. The dots mark the positions, in $\left(\Delta \phi_{i j}, v\right)$ space, of every point in the spatially degraded cube (we also show the variation of mean and mean plus/minus $1-\sigma$ of $\Delta \phi_{i j}$ at each $v$-indicated by the dash-dot and solid lines respectively). Of these points, about $10 \%$ lie between 3 and $8 \mathrm{mHz}$ with a net cross-power greater than, or equal to, ten. This latter set are indicated by triangles and their size indicates the net crosspower, the quantity used to weight the linear fit. The solid line indicates the fit to the triangles and has a gradient of $7.4 \pm 0.8^{\circ} \mathrm{mHz}^{-1}$.

1700-1550 ̊, and 1600-1550 ̊ respectively. We see that there is a variation of $M_{\Delta \phi}$ from about $0^{\circ} \mathrm{mHz}^{-1}$ in the network regions to about $10^{\circ} \mathrm{mHz}^{-1}$ in the farthest reaches of the internetwork. As noted above, for this degree of spatial binning, there is an associated mean error in $M_{\Delta \phi}$ of $\pm 1^{\circ} \mathrm{mHz}^{-1}$. We note that, at full TRACE spatial resolution, the error is $\sim \pm 10^{\circ} \mathrm{mHz}^{-1}$ and it is straightforward to see that any spatial pattern is lost in the noise attributed to the poorly constrained fit (as mentioned above). As a test of our methodology we have performed a "closure test" (cf. Sect. 5 of Krijger et al. 2001) involving the comparison of the 1700-1550 map to the sum of the other two; the resulting difference should be zero. For our level of approximation, using $M_{\Delta \phi}$ as a measure of the phase-difference variation, we are close to zero (mean difference of $0.5 \pm 0.4^{\circ} \mathrm{mHz}^{-1}$ ).

As in Sect. 2, we notice that there is a striking correspondence between regions of low $M_{\Delta \phi}$ and the lower $\beta$ TH contours, predominantly in the network regions. In the internetwork regions, far away from the network elements, we see the largest values of $M_{\Delta \phi}$. In Fig. 7, as in Fig. 3, we show the correlation histograms of $M_{\Delta \phi}$ for each of the bandpass pairs and $\beta \mathrm{TH}$. Again, the two correlations discussed above are evident in each panel. We note this correspondence here and leave a brief discussion for the following section.

\section{Discussion}

We have shown that there is a distinct correlation between the inferred solar plasma topography, the phase-differences between and suppression of oscillations in different levels of the 

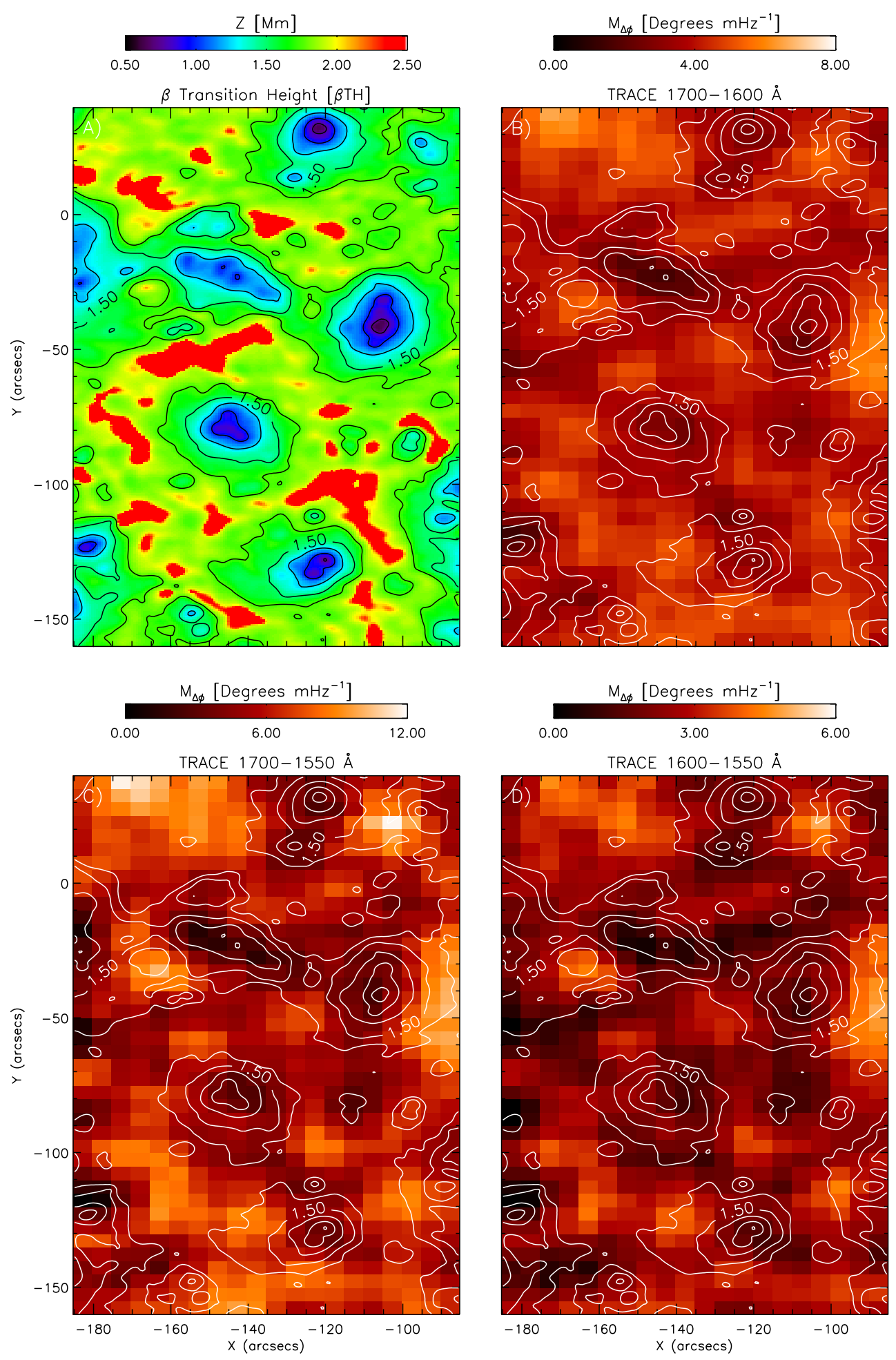

Fig. 6. As in Fig. 2, in panel A, we show the model-derived altitudes at which the plasma- $\beta$ is of order unity in the TRACE field of view. In panels B through D we show the fitted phase-difference gradient $\left(M_{\Delta \phi}\right)$ maps as discussed in the text. Again, we note more than a passing correspondence between the overplotted contours and the darker regions of the $M_{\Delta \phi}$ maps.

outer solar atmosphere. The suppression of the TRACE signal, near network elements, is reported by Krijger et al. (2001) but the extension of the suppression far beyond the network region and its connection to the $\beta \mathrm{TH}$ was not made. The fact that this extension of the suppressed power is not observed in the lower photosphere (the MDI time-series) we interpret as evidence of interaction between oscillations and the $\beta \mathrm{TH}$ "canopy", i.e., the two-dimensional analogue of the 3-min oscillation suppression reported by McIntosh et al. (2001).

We acknowledge that there are drawbacks associated with the use of a potential field extrapolation and the incorporation of the VAL3C atmospheric model in building the plasma 


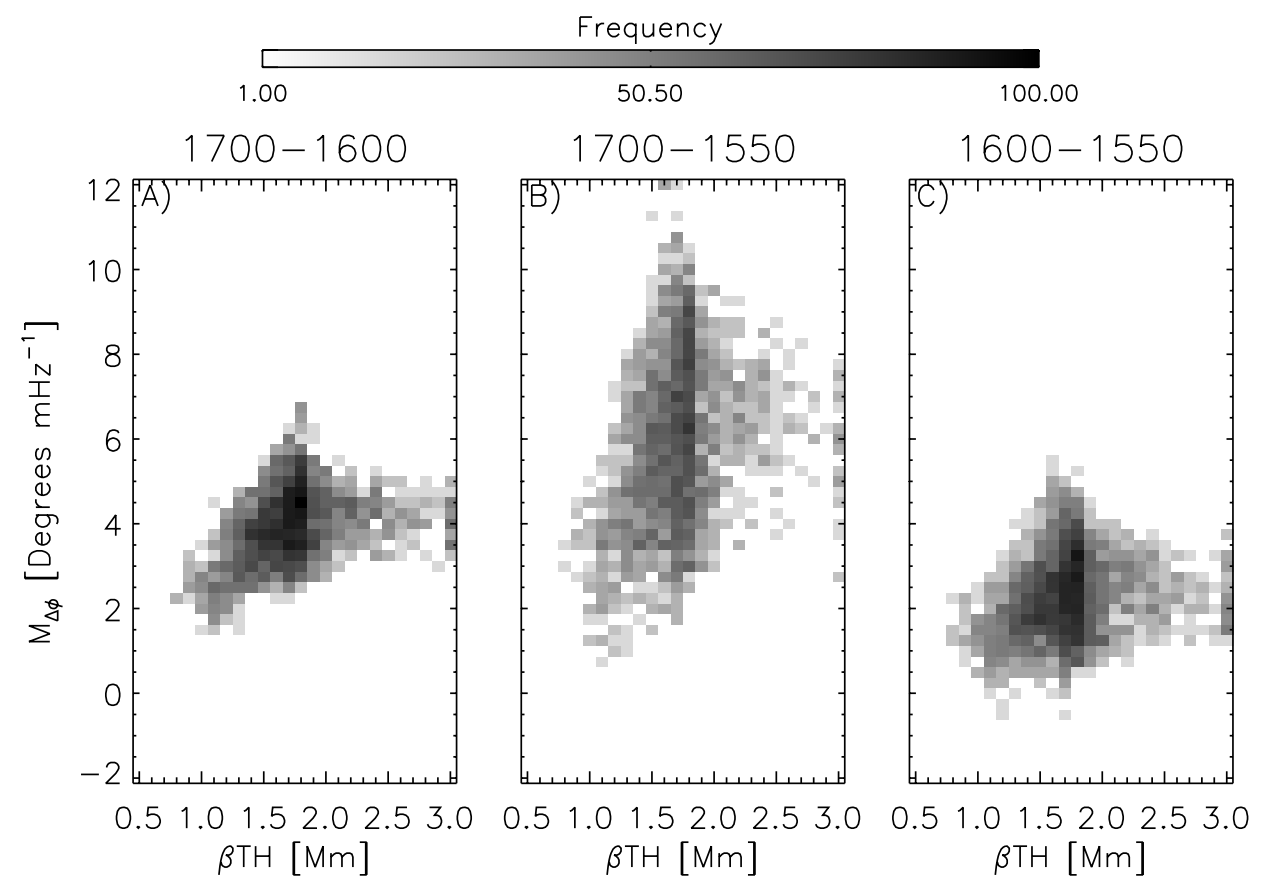

Fig. 7. The two-dimensional histograms of phase-difference gradient $\left(M_{\Delta \phi}\right)$ and $\beta$ TH derived from panels B through D of Fig. 6. The colour scale in each panel indicates the number of image pixels with that particular combination of $M_{\Delta \phi}$ and $\beta$ TH. As for Fig. 3, we see that, in each panel, there are two distinct correlations present. The "tail" to the lower-left indicating the correlation of low $M_{\Delta \phi}$ and low $\beta \mathrm{TH}$ and the broader $M_{\triangle \phi}$ correlation of the "canopy" at a $\beta \mathrm{TH}$ of around $1.75 \mathrm{Mm}$. In this case, the "resolution" of the histograms are degraded compared to those of Fig. 3 but this is due to the spatial degradation necessary to compute $M_{\Delta \phi}$ from the full resolution TRACE image.

topography (e.g., concentrated flux regions). For example, the slight discrepancy between the TRACE formation heights and those attributed from the $\beta \mathrm{TH}$ can most likely be attributed to the application of the VAL3C to regions of the atmosphere where it would typically be invalid. In any further analysis the use of a lookup table to associate model atmosphere type and, say, magnetic field strength may provide more accurate predictions of the $\beta \mathrm{TH}$ interface.

In a future paper (McIntosh et al. 2003) we investigate, in depth, the spatial patterns of $M_{\Delta \phi}$ presented in the panels of Fig. 6. To this end we consider the two significant components that we have mentioned in Sect. 2.1, discussed at greater length in Lites et al. (1982); Deubner \& Fleck (1990), that can produce these patterns in imaging observations:

- The role of inferred plasma topography on the observed $M_{\Delta \phi}$ patterns in terms of identifying the specific magnetoatmospheric wave-modes present at those locations through the spatial variations in phase-speed. Multi-dimensional magnetohydrodynamic simulations are the key to decoupling and understanding these wave-modes, as discussed in Rosenthal et al. (2002); Bogdan et al. (2002, 2003).

- The amount by which the $M_{\Delta \phi}$ pattern depends on the amount of vertical overlap of the formation heights of the observed TRACE continua. The greater the degree of overlap, the smaller the value of $M_{\Delta \phi}$, as would most likely be the case in the network plasma. Conversely, in the farthest reaches of the internetwork plasma we might expect the least overlap of the bandpasses. In this case, detailed radiation transfer calculations, like those presented in Carlsson \& Stein (2002), of these bandpasses are required to evaluate the exact degree of overlap for specific physical conditions.

The work presented in this paper (and in McIntosh et al. 2001; McIntosh \& Judge 2001; Rosenthal et al. 2002; Bogdan et al. 2002 , 2003) demonstrates that understanding the position and role of the $\beta \mathrm{TH}$ may provide a critical link into the understanding of wave mode conversion, dissipation and ultimately the heating mechanism of the chromosphere, maybe even the ambient solar corona itself. However, for the time being, the investigation of these effects will be limited to the complex, though not impossible (cf. JTW), combination of spectroscopic slit (SUMER) and passband imaging (TRACE) observations which lack Doppler velocity information. Indeed, that will be the case until we are able to perform some form of imaging spectroscopy. Such a platform will allow the study of multiple line profiles simultaneously, spanning the vertical domain of the solar atmosphere in a less discrete fashion. This advance will accurately allow the diagnosis and mapping of the important region where the plasma- $\beta$ is of order unity.

Acknowledgements. SWM acknowledges the support of a External Fellowship from the European Space Agency at GSFC and the European Solar Magnetometry Network (ESMN) under contract ERBFMRXCT980190. The authors wish to thank Guiseppe Severino, Mark Rast and Karel Schrijver for their careful reading of the manuscript and the comments that have greatly improved its content and clarity. SWM also wishes to acknowledge the support and assistance of Tom Bogdan, Mats Carlsson and Viggo Hansteen in the work that has lead up to this publication. 


\section{References}

Bodgan, T. J., Rosenthal, C. S., Carlsson, M., et al. 2002, Astron. Nachr., 323, 196

Bodgan, T. J., Rosenthal, C. S., Carlsson, M., et al. 2003, ApJ, March 2003, submitted

Carlsson, M., \& Stein, R. F. 2003, ApJ, 572, 626

Deubner, F.-L., \& Fleck, B. 1990, A\&A, 228, 506

Fleck, B., Domingo, V., \& Poland, A. I. 1995, The SOHO Mission (Dordrecht: Kluwer)

Handy, B. N., Acton, L. W., Kankelborg, C. C., et al. 1999, Sol. Phys., 187,229

Jain, R., \& Haber, D. 2002, A\&A, 387, 1092

Judge, P. G., Tarbell, T. D., \& Wilhelm, K. 2001, ApJ, 554, 424

Krijger, J. M., Rutten, R. J., Lites, B. W., et al. 2001, A\&A, 379, 1052
Lites, B. W., Chipman, E. G., \& White, O. R. 1982, ApJ, 253, 367

McIntosh, S. W., Bogdan, T. J., Cally, P. S., et al. 2001, ApJ, 548, L237

McIntosh, S. W., \& Judge, P. G. 2001, ApJ, 561, 420

McIntosh, S. W., Fleck B., Judge, P. G., \& Bogdan, T. J. 2003, in preparation

Metcalf, T. R., Jiao, L., McClymont, A. N., et al. 1995, ApJ, 439, 474

Rosenthal, C. S., Bogdan, T. J., Carlsson, M., et al. 2002, ApJ, 564, 508

Scherrer, P. H., Bogart, R. S., Bush, R. I., et al. 1995, Sol. Phys., 162, 129

Title, A. M., Topka, K. P., Tarbell, T. D., et al. 1992, ApJ, 393, 782

Vernazza, J. E., Avrett, E. H., \& Loeser, R. 1981, ApJS, 45, 635

Wilhelm, K., Curdt, W., Marsch, E., et al. 1995, Sol. Phys., 162, 189 